\title{
Design and Analysis of a Vibration-Driven Electromagnetic Energy Harvester Using Multi-Pole Magnet
}

\author{
Ahmed Munaz and Gwiy-Sang Chung ${ }^{+}$
}

\begin{abstract}
This paper presents the design and analysis of a vibration-driven electromagnetic energy harvester that uses a multi-pole magnet. The physical backgrounds of the vibration electromagnetic energy harvester are reported, and an ANSYS finite element analysis simulation has been used to determine the different alignments of the magnetic pole array with their flux lines and density. The basic working principles for a single and multi-pole magnet are illustrated and the proposed harvester has been presented in a schematic diagram. Mechanical parameters such as input frequency, maximum displacement, number of coil turns, and load resistance have been analyzed to obtain an optimized output power for the harvester through theoretical study. The paper reports a maximum of $1.005 \mathrm{~mW}$ of power with a load resistance of $1.9 \mathrm{k} \Omega$ for 5 magnets with 450 coil turns.
\end{abstract}

Keywords : Energy Harvester, Electromagnetic, Low Frequency, Multi-pole Magnet, Vibration

\section{INTRODUCTION}

Research and investments in green energy are currently garnering a great deal of interest because of the limited amount of conventional resources like oil, coal, gas, fuel, etc. In addition to the scarcity, these resources require large power production facilities, and they pollute the environment too. To overcome these limitations, alternative sources like solar, wind, thermal and ocean waves can be taken into consideration[1]. But, the production of power using these sources is costly, complex and large in scale. On the other hand, the use of various small-scale portable devices such as $\mathrm{mp} 3$ players, mobile phones and wireless sensors has increased tremendously. To power these small portable devices, conventional resources and large-scale alternative sources do not seem suitable. While high-power batteries like alkaline(nonrechargeable), nickel metal hydride and lithium ion(rechargeable) are available, such batteries need to be charged regularly and have a limited life cycle after which they must be disposed of. Also, these conventional batteries involve chemical reactions that pollute the environment. Considering all of these limitations, a vibration source seems quite suitable for powering smallscale portable devices because of its low cost, smaller scale

School of Electrical Engineering, University of Ulsan

${ }^{+}$Corresponding author: gschung@ulsan.ac.kr

(Received : Mar. 12, 2012, Revised : Apr. 9, 2012, Accepted : Apr. 21, 2012) and easy availability of the source materials in the environment[2]. Various types of vibration-based energy harvesters such as electromagnetic, piezoelectric and electrostatic devices have been investigated[3]. Among these, electromagnetic harvesters seem appropriate because of their simple structure and low resonance frequency. Various electromagnetic energy harvesters using a single magnet have been investigated[4, 5]. However, the power from these single magnet energy harvesters is very low and insufficient to power today's electronic devices.

To solve this problem and to increase the efficiency of the harvester, multi-pole magnets have been used by some groups[6-9]. The output voltage and power were increased in these cases, but this was accompanied by an undesirable increase in harvester size. For example, Beeby et al.[2] present an electromagnetic harvester with a volume of 0.15 $\mathrm{cm}^{3}$ that produces $46 \mu \mathrm{W}$ of power in a resistive load of 4 $\mathrm{k} \Omega$. The generator uses four magnets arranged on an etched cantilever with a resonance frequency of $52 \mathrm{~Hz}$. Cepnik et al.[8] show a harvester capable of producing average output power of $20.6 \mathrm{~mW}$ at $50 \mathrm{~Hz}$ frequency. This generator use six magnets on a spring as a moving mass and the harvester volume is $10.8 \mathrm{~cm}^{3}$ with excitation of $1.03 \mathrm{~g}$.

The present study is focused on the design and analysiss of a multi-pole magnet energy harvester capable of producing ample power within a limited volume for a particular magnetic pole alignment. 


\section{DESIGN}

\subsection{Physical Background}

A vibration-driven electromagnetic generator is a second order spring mass system. Fig. 1(a) shows a schematic diagram of a basic vibration-driven electromagnetic energy harvester. The harvester consists of an inertial mass ' $m$ ' (which is a magnet) suspended with a spring of spring constant ' $k$ '. Energy losses within the harvester are represented by damping co-efficient ' $d$ '. The losses are comprised of parasitic damping and electrical damping[10].

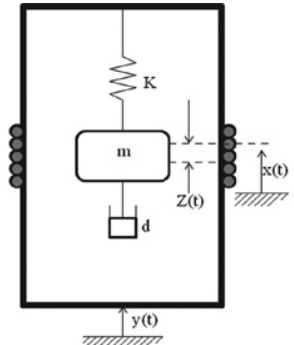

(a)

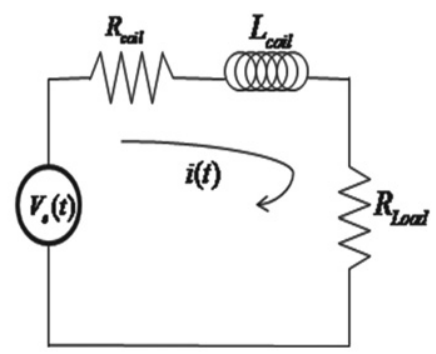

(b)
Fig. 1. (a) Schematic diagram of a basic electromagnetic energy harvester and (b) equivalent electrical circuit.

When the harvester starts to oscillate with vibration displacement of $y(t)$, the mass ' $m$ ' starts to move up and down. The movement of the mass is represented by $x(t)$. Then the differential equation of the second order spring mass system is:

$$
\begin{aligned}
& m \ddot{x}(t)+d[\dot{x}(t)-\dot{y}(t)]+k[x(t)-y(t)]=0 \\
& m \ddot{Z}(t)+d \dot{Z}(t)+k Z(t)=-m \ddot{y}(t)
\end{aligned}
$$

where, $Z(t)=x(t)-y(t)$.

Here, $Z(t)$ is the relative displacement between the moving mass and harvester housing. The harvester works as a transducer which converts the mechanical energy to desired electrical voltages. The corresponding electrical representation is given in Fig. 1(b). Here, the relative motion between the mass and coil creates rates of change of flux which produce voltage $V e(t)$ with respect to the load. The equivalent equation is:

$$
V_{e}=i R_{\text {coil }}+i L_{\text {coil }}+i R_{\text {load }}
$$

According to Faraday's law of induction, when a magnet oscillates by an external force, the coil cuts the flux line of the magnet, thus producing voltage. According to the law,

$$
V_{c o i l}=-N \frac{d \phi}{d t}
$$

where $N$ is the number of turns and $d \emptyset / d t$ is the rate of change in flux. By varying $d \varnothing / d t$, the total induced voltage can be changed[7].

\subsection{ANSYS Simulation}

Initially, an ANSYS 12.1 finite-element analysis is performed to determine the magnetic flux lines and flux densities of magnets with the same volume but different pole arrays, as shown in Figs. 2 - 4.

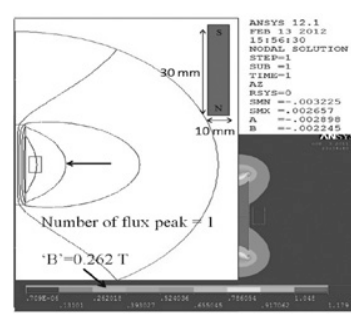

(a)

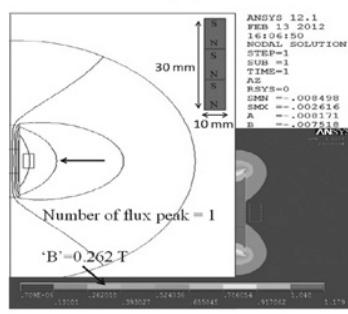

(c)

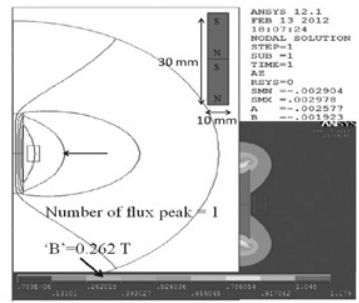

(b)

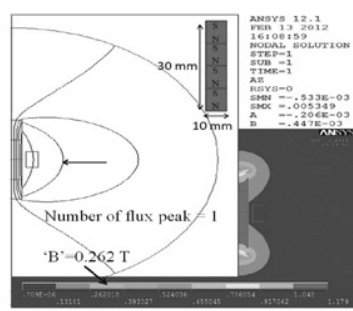

(d)
Fig. 2. ANSYS simulation of magnetic pole alignment towards the $Y$-axis (a) 1-magnet ' $\mathrm{B}$ ' $=0.262$ (T), (b) 2-magnet ' $\mathrm{B}$ '=0.262 (T), (c) 3-magnet ' $\mathrm{B}$ '=0.262 (T), (d) 4-magnet 'B'=0.262(T).

Fig. 2 shows the pole alignment of the magnetic flux lines and corresponding flux density for 1-4 magnets. For a single magnet, one flux peak with a flux density of $0.262 \mathrm{~T}$ can be seen. Next, the magnet is separated into two, three, and four parts, in a way that the north pole of each magnet is attracted to the south pole of another magnet while keeping the aspect ratio the same. In this case, a flux 
density of $0.262 \mathrm{~T}$ is observed for two, three, and four magnets, with only one flux peak. Therefore, if this type of magnetic pole alignment is used in an energy harvester, the total efficiency will be the same.

Fig. 3 shows the magnetic pole alignment towards the Yaxis, where the south poles of each magnet is connected to each other and vice versa. As shown in Figs. 3(a)-3(f), the flux densities are $0.262 \mathrm{~T}, 0.3224 \mathrm{~T}, 0.3133 \mathrm{~T}, 0.3085 \mathrm{~T}$, $0.2960 \mathrm{~T}$, and $0.2934 \mathrm{~T}$ for $1-6$ magnets respectively.

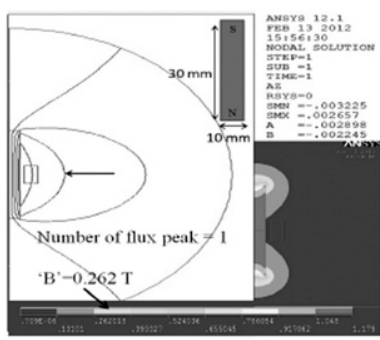

(a)

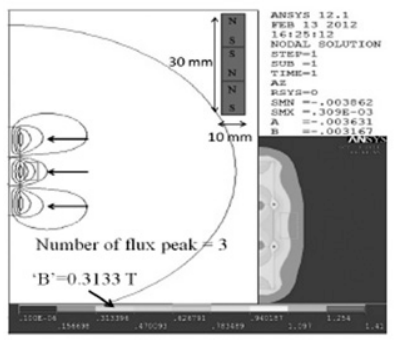

(c)

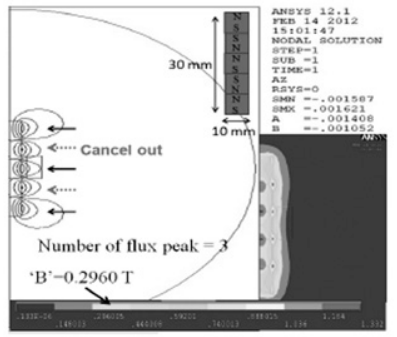

(e)

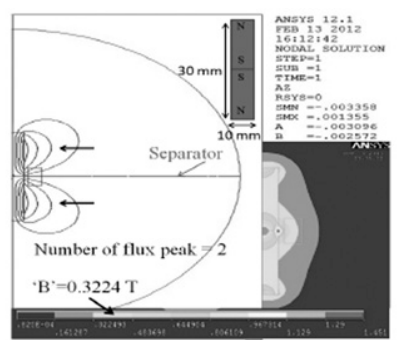

(b)

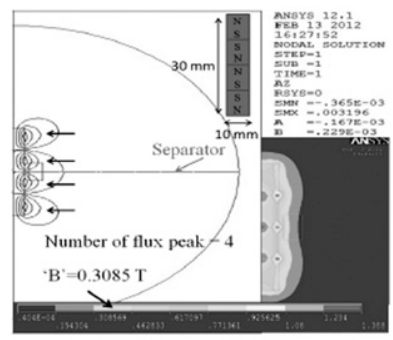

(d)

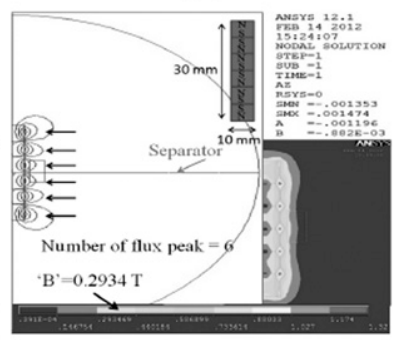

(f)
Fig. 3. ANSYS simulation of magnetic pole alignment towards the $\mathrm{Y}$-axis for (a) 1-magnet ' $\mathrm{B}$ ' $=0.262$ (T), (b) 2-magnet ' $\mathrm{B}$ '=0.3224 (T), (c) 3-magnet ' $\mathrm{B}$ ' $=0.3133$ (T), (d) 4-magnet 'B'=0.3085 (T), (e) 5-magnet ' $\mathrm{B}$ '=0.2960 (T), (f) 6-magnet 'B'=0.2934 (T).

The flux density of two magnets is increased compared to that of the single magnet. But for 3-6 magnets in Figs. 3(c)-3(f), the flux densities are almost the same. Figs. 3(b), $3(\mathrm{~d})$, and 3(f) depicted that, for 2, 4, and 6 magnets, there is a flux line which is separating these regions into two parts. These separators in the magnet prevent the flux peaks from cancelling each other out when goes through a coil. So the numbers of flux peaks are 2, 4, and 6 respectively. But for 1,3 , and 5 magnets there is no such separator. As a result, some of the flux peaks become neutralized. Therefore the number of flux peaks is 1,3 , and 3 for single, three, and five magnets, respectively. From this analysis, all even number magnets $(2,4$, and 6$)$ show a separator that creates two regions in the active area, thus the change in flux and output increases, whereas for 3 and 5 magnets, the output remains almost the same. But it is not easy to connect south-south and north-north poles of a magnet directly in this second design. Therefore keeping a small gap between these magnets is necessary. Many groups have tried to keep this gap using air, soft-magnets, or other materials[68]. But in these cases, the aspect ratio increases while comparing one magnet with another one. Also, the height of the harvester may vary due to the change of height in the moving magnet.

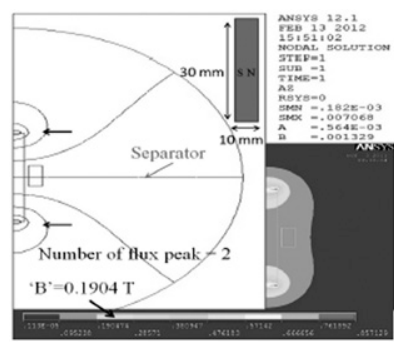

(a)

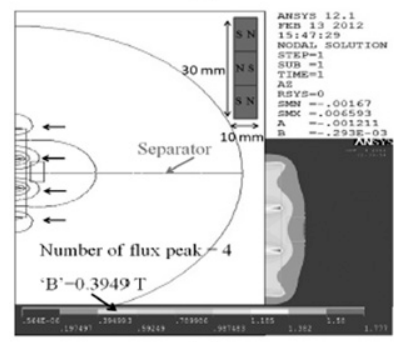

(c)

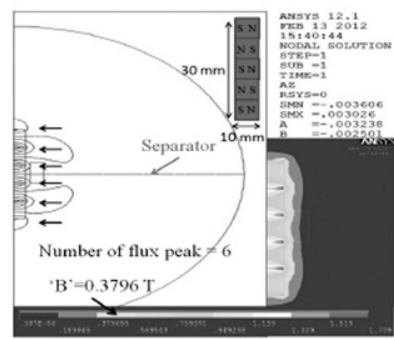

(e)

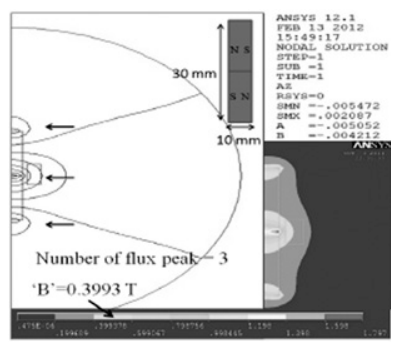

(b)

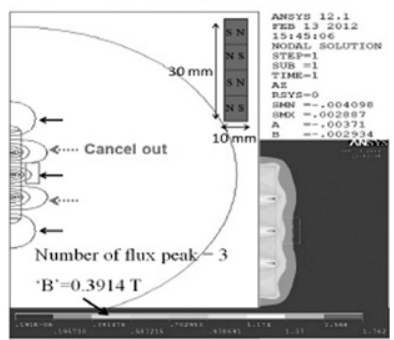

(d)

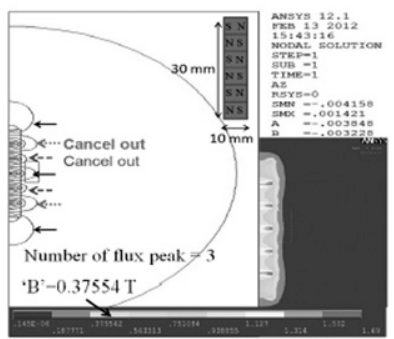

(f)
Fig. 4. ANSYS simulation of magnetic pole alignment towards the $X$-axis (a) 1-magnet ' $B$ ' $=0.1904$ (T), (b) 2-magnet ' $\mathrm{B}$ ' $=0.3993$ (T), (c) 3-magnet ' $\mathrm{B}$ ' $=0.3949$ (T), (d) 4-magnet 'B'=0.3914 (T), (e) 5-magnet ' $\mathrm{B}$ ' $=0.3796$ (T), (d) 6-magnet 'B'=0.37554 (T). 
Fig. 4 shows the magnetic pole alignment towards the Xaxis for each magnet, where it is very easy to connect different pole arrays. In these pole arrays, higher flux density is achievable, and the problem of connecting the same poles has been overcome(it is not necessary to keep the gap between each magnet, so it is possible to maintain the aspect ratio at the same value). Figs. 4(a)-4(f) show flux densities of $0.1904 \mathrm{~T}, 0.3993 \mathrm{~T}, 0.3949 \mathrm{~T}, 0.3914 \mathrm{~T}$, $0.3796 \mathrm{~T}$, and $0.37554 \mathrm{~T}$ for 1-6 magnets respectively. The active separator can be seen in Figs. 4(a), 4(c), and 4(e) for single, triple, and five magnets.

Because of the separator, the numbers of active flux peaks are 2, 4, and 6. But as the moving magnets are separated into 2, 4 and 6 parts in Figs. 4(b), 4(d), and 4(f), there are no such separator in the region. As a result, when the magnet moves throughout a coil, some of the flux peaks become cancelled out. Therefore the numbers of flux peaks are three for 2,4 , and 6 magnets. Thus the $d \varnothing / d t$ in total remains almost the same. From this analysis, all odd numbers of magnets in Fig. 4 show a separator, which creates two regions in the active area. Thus the rates of change in the flux and output increase.

By analyzing the flux lines and density of these magnets in Figs. 3-4, it can be seen that the flux line area reduces as the number of magnets increases. So if the magnet is divided into more parts, then there will be more flux peaks. But because of the more compressed area, flux lines will not be able to cut the entire coil. As a result, the total efficiency of the harvester will be reduced. To get high output, the coil of the harvester should be positioned as close as possible to the magnet. Among these three designs, the third magnetic pole alignment has been proposed as a moving magnet for the harvester due to higher flux density.

\subsection{Variations of flux and voltage of a multi-pole magnet}

A simplified qualitative illustration of the full mechanical cycle of a single magnet is shown in Fig. 5(a), along with the time period $(\mathrm{T})$ in the $\mathrm{X}$-axis and corresponding flux and voltage cycle in the $\mathrm{Y}$-axis. When the magnet starts to move vertically from its initial position, there is an increase in the rate of change of the flux. At the center of the coil, the flux reaches a maximum. Then, the magnet moves further downward and the flux starts to decrease. The flux reaches its minimum value when the magnet is opposite to its initial position. Again, the magnet starts to move upward and the same flux change occurs. Thus, in a single full mechanical cycle, two flux cycles occur for each magnet. In the meantime, four voltage cycles are induced by the corresponding fluxes. According to Faraday's law of induction, this voltage is in opposition to the corresponding fluxes[7]. Thus, in one full mechanical cycle of a single magnet, two corresponding flux cycles and four voltage cycles are produced.

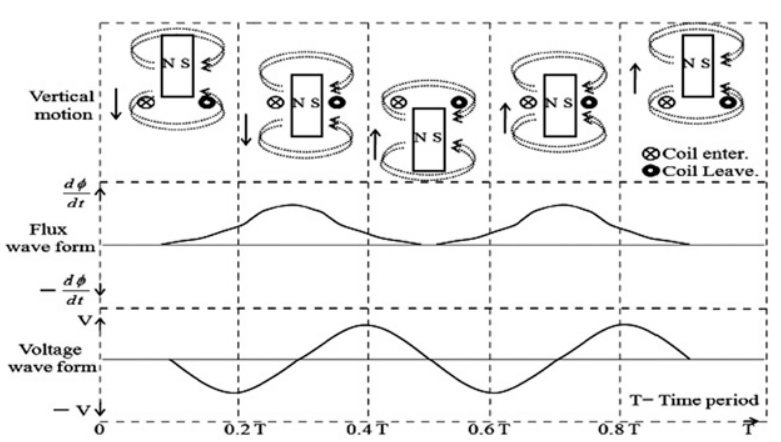

(a)

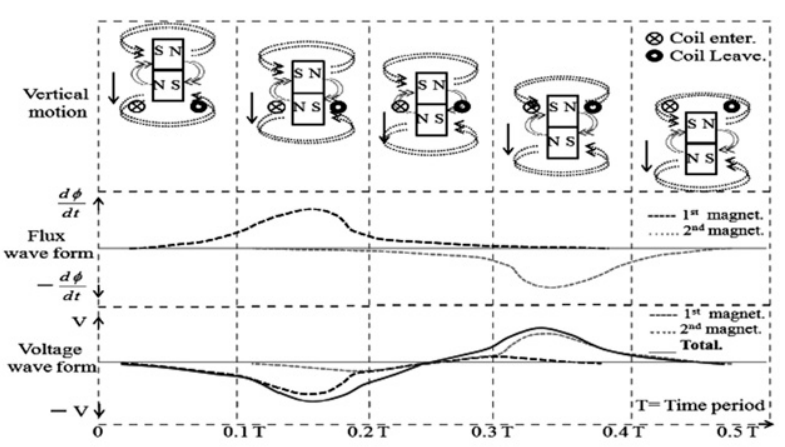

(b)

Fig. 5. Simple qualitative illustration of mechanical motion for (a) a single magnet(full cycle) and (b) two magnets(half cycle).

Half mechanical motion for two magnets of the same volume but with opposite polarities is illustrated qualitatively in Fig. 5(b), where the time period (T) is in the $\mathrm{X}$-axis, and corresponding flux and voltage cycle is in the $\mathrm{Y}$-axis. In one half of the mechanical cycle, each magnet creates single flux cycle. The two magnets have opposite polarity, so that the flux cycles are also opposite. Thus, one half of a mechanical cycle produces two fluxes and four corresponding voltage cycles, whereas a single magnet would require a full cycle to achieve these same results. So, a full mechanical cycle with two magnets results in twice the flux and twice the corresponding voltage changes. If an induction coil is connected in series, the resulting voltage will obviously increase. For three and four magnets(half of 
a mechanical cycle), the corresponding flux cycles will be three and four, and the voltage cycles will be six and eight, respectively[7].

It should be noted that the flux density and voltage through the coil shown in the figure will not be the same as in a real system. The exact performance requires more precise modeling. The flux density and voltage could decrease due to damping or they could increase due to use of a high grade magnet. These figures do not represent precise parameters; they simply explain the basic working steps for single and multi-pole magnets.

\subsection{Harvester schematic diagram}

Fig. 6 shows a schematic diagram of an optimized vibration-driven electromagnetic energy harvester. The proposed structure is composed of a rectangular housing made of acrylic glass, a copper coil and an active mass. The third magnetic pole design from the simulation is proposed for the active mass in the harvester, which is a rectangular shaped NdFeb magnet. Furthermore, similar magnets are proposed to create magnetic springs for the moving mass at each end of the housing. Smaller coil height provides more rate of change in flux $d \varnothing / d t$, thus more output is achievable[10]. Therefore a coil with height of $7 \mathrm{~mm}$ is proposed for the harvester.

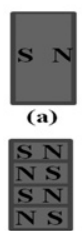

(d)

Moving ma (a) 1 - magnet $(30 \times 10 \times 5) \mathrm{mm}$ (b) 2 - magnet $(15 \times 10 \times 5) \mathrm{mm}$ (c) 3-magnet $(10 \times 10 \times 5) \mathrm{mm}$ (d) 5 - magnet $(6 \times 10 \times 5) \mathrm{mm}$ (f) 6 - magnet $(5 \times 10 \times 5) \mathrm{mm}$

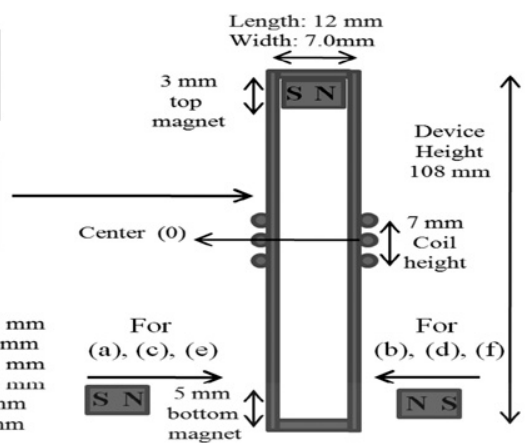

Fig. 6. Schematic diagram of a multi-pole energy harvester.

\section{ANALYTICAL STUDY}

In order to predict practical performances of the devices, an analytical study including damping and other parameters is conducted. The damping includes parasitic or mechanical and electromagnetic losses. The parasitic damping represents loss mechanisms, such as air damping, friction between housing and the moving mass, etc. The electromagnetic damping represents the mechanism through which electrical power is extracted from the system[10]. In a system, maximum efficiency can be obtained by decreasing the parasitic damping and increasing the electromagnetic damping. From Eq. (2), the relative displacement of a system $Z(t)$ can be written as the steady state response of the seismic mass as:

$$
Z(t)=Z_{0} \cos (w t-\phi)
$$

where, $Z_{0}$ is the amplitude of the mass $m$, and $w$ is the frequency of the system. $\varnothing$ and $Z_{0}$ can be obtained from the following equation:

$$
\begin{aligned}
& \phi=\tan ^{-1}\left(\frac{d w}{k-m w^{2}}\right), \\
& \text { and } Z_{0}=\frac{m w^{2}}{\sqrt{\left(k-m w^{2}\right)^{2}+(d w)^{2}}} Y_{0}
\end{aligned}
$$

Here, $k$ is the spring constant, $d$ is the harvester damping and $Y_{0}$ is the vibration amplitude[11]. The most important factor for this analytical study is the amount of power produced by the generator. The instantaneous power $p(t)$ transferred from the source to the micro generator is given as:

$$
p(t)=-[k Z(t)+d \dot{Z}(t)] \dot{y}(t)
$$

Eq. (6) can be modified as:

$$
p_{g e n}=\frac{m Y_{0}^{2} w^{3} z r^{3}}{\left[\left(1-r^{2}\right)^{2}+(2 z r)^{2}\right]}
$$

where frequency ratio $r=w / w_{n}$, natural frequency $w_{n}=\sqrt{k / m}$, and $z$ represents total damping of the entire system[11]. Maximum power can be achieved by further modifying Eq. (7) as:

$$
P_{\max }=\frac{m Y_{0}^{2} w^{3}}{4 z}
$$

Using eq. (8), maximum power versus input vibration amplitude is analyzed and is shown in Fig. 7. The analysis shows that amplitude is proportional to the power.

Fig. 8 shows an increase in power with increase in input frequency. As the frequency increases, the corresponding power also increases. However, increasing the frequency is not always practical because ambient vibration is very 
low(below $10 \mathrm{~Hz}$ ), and the harvester needs to adjust to this low vibration. Frequency must be optimized for the harvester operation. Eq. (8) can further be modified as:

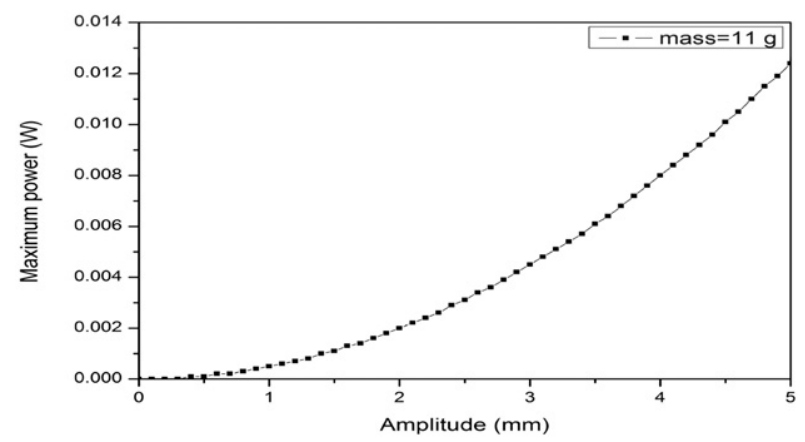

Fig. 7. Maximum power versus amplitude.

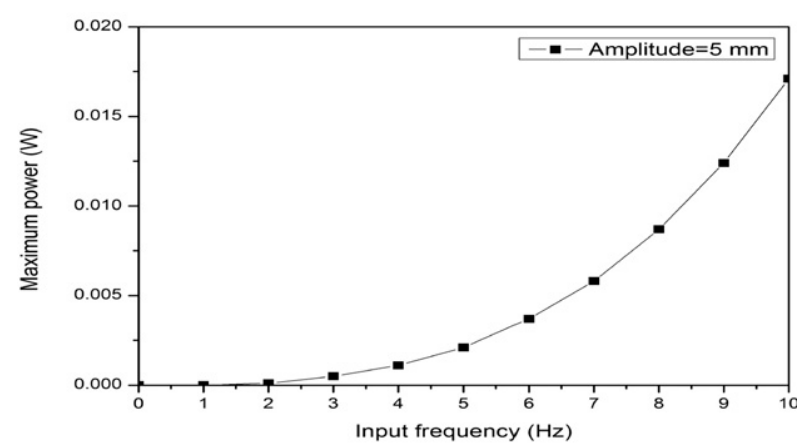

Fig. 8. Maximum power versus input frequency.

$$
P_{a v g}=\left(y^{2} w^{2} s^{2}\right) /\left(8 z_{t}^{2}\left(R_{l}+R_{c}\right)\right.
$$

where, $z_{t}=z_{e}+z_{m}$

Here, $Z_{m}$ is the mechanical damping and $Z_{e}$ is the electromagnetic damping and as follows:

$$
\begin{aligned}
& Z_{m}=d /(2 m w) \\
& z_{e}=s^{2} / 2 m w\left(R_{l}+R_{c}\right), \text { and } s=N B l
\end{aligned}
$$

Here, $N$ is the number of turns in the coil, $l$ is the length of the coil, $B$ is the magnetic flux density and $R_{c}$ and $R_{l}$ are coil resistance and load resistance, respectively. Using eq. (9), some parameters for 1-6 magnets are analyzed in Figs. 9 - 10.

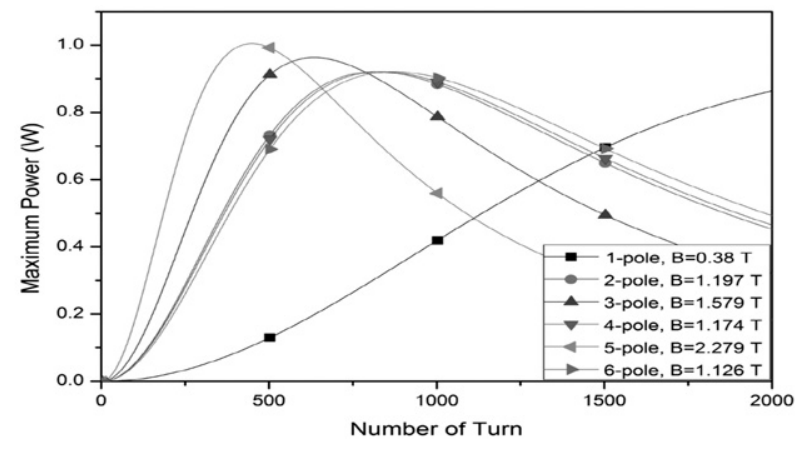

Fig. 9. Maximum power vs. coil number of turn.

Fig. 9 shows the calculated power versus the numbers of coil turns for all of the studied magnets. As shown in Fig. 9, a maximum power of $1.005 \mathrm{~mW}$ is obtained at 450 coil turns for five pole magnets. As the turn number increases for multiple magnets, the more condensed flux lines could not cut the outer coil. However, an increase in turn number leads to an increase in the coil resistance, which results in a rapid reduction in power. But for single magnet, even though flux density is low, the area of the flux lines is more scattered. So a single magnet can cut the most outer flux lines as the coil turns increase. But, the total efficiency is low compared to that in the multiple magnet cases.

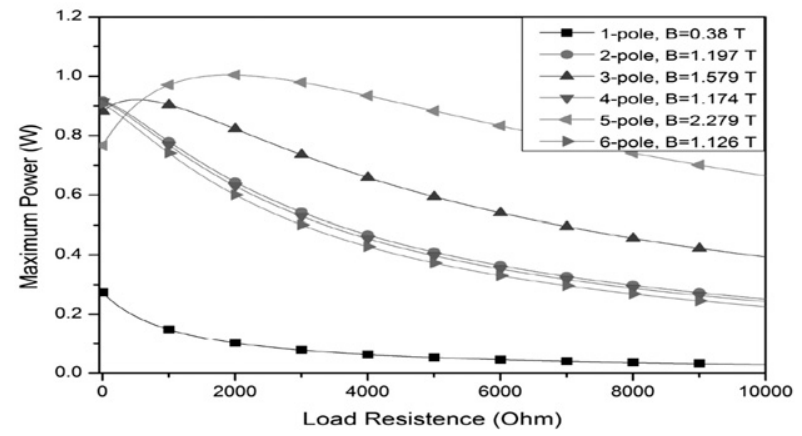

Fig. 10. Maximum power vs. Load resistance.

Fig. 10 shows the calculated power versus load resistance for 1-6 magnets. The output power for 5 magnets reaches its maximum value of $1.005 \mathrm{~mW}$ at a load resistance of $1.9 \mathrm{k} \Omega[11]$.

In the analytical section, all the parameters are the same except for only dividing the active mass into more pieces, which allows the rate of flux $d \varnothing / d t$ to change more quickly. The power of 2, 4, and 6 magnets are almost the same because of the number of flux peaks which are the same too. 


\section{CONCLUSIONS}

In this work, a vibration-driven electromagnetic energy harvester using a multi-pole magnet is designed and analyzed. An ANSYS simulation has been used to study the different pole alignments of the magnet. In the analytical section, different damping factors and maximum power are demonstrated using different parameters in MATLAB. The analysis shows that a maximum of 1.005 $\mathrm{mW}$ of power could be achievable with a load resistance of $1.9 \mathrm{k} \Omega$ for 5 magnets with 450 coil turns.

\section{ACKNOWLEDGEMENTS}

This work was supported by the Next Generation Military Battery Research Center; Program of the Defense Acquisition Program Administration, the Agency for Defense Development, and the Korea Research Foundation Grant funded in 2011 by the Korean Government, which was conducted by the Ministry of Education, Science and Technology(No. 2011-0013831).

\section{REFERENCES}

[1] S. A. Jacobson and A. H. Epstein, "An informal survey of power MEMS”, Proc. of int. Symp. on MicroMech. Eng., vol. 12, pp. 513-519, 2003.

[2] S. P. Beeby, R. N. Torah, M.J. Tudor, P. G. Jones, T. O. Donnell, C. R. Saha, and S. Roy, "A micro electromagnetic generator for vibration energy harvesting", $J$. Micromech. Microeng., vol. 17, pp.1257-1265, 2007.

[3] T. Sterken, K. Baert, V. Hoof, Puers, G. Borghs, and
P. Fiorini, "Comparative modeling for vibration scavengers”, Sensors, vol. 3, pp. 1249- 1252, 2005.

[4] S. C. Yuen, J. M. Lee, W. J. Li, and P. H. Leong, “An AA-sized vibration-based micro-generator for wireless sensors", Proc. of IEEE Conf. on Pervasive Comput., vol. 6, pp. 64-72, 2007.

[5] B. P. Mann and N. D. Sims, "Energy harvesting from the nonlinear oscillation of magnetic levitation", $J$. of Soun \& Vibration, vol. 319, pp. 515-530, 2009.

[6] V. B. Thomas and G. Troster, "Design and optimization of a linear vibration-driven electromagnetic micro-power generator", Sens. Actu. A, vol. 135, pp. 765-775, 2007.

[7] S. Cheng and D. P. Arnold, "A study of a multi-pole magnetic generator for low-frequency vibration energy harvesting", J. Micromech. Microeng., vol. 20, no. 2, pp. 25015(1)- 25015(10), 2010.

[8] C. Cepnik, O. Radler, S. Rosenbaum, T. Strohla, and U. Wallrabe, "Effective optimization of a electromagnetic energy harvester through direct computation of the electromagnetic coupling', Sens. Actu. A, vol. 167, pp. 416-421, 2011.

[9] Z. Dibin , S. P. Beeby, M. J. Tudor, and N. R. Harris, "A planner electromagnetic vibration energy harvester with a halbach array", Proc. of IEEE Conf. on Power MEMS., vol. 11, pp. 347-350, 2011.

[10] T. O. Donnell, C. Saha, S. Beeby, and J. Tudor, "Scaling effects for electromagnetic vibration power generator", Microsyst. Technol., vol. 13, no. 11-12, pp. 1637-1645, 2006.

[11] N. Awaja, D. Sood, and T. Vinay, "Design and analyses of electromagnetic micro-generator", Sens. Trans., vol. 103, pp. 109-121, 2009. 


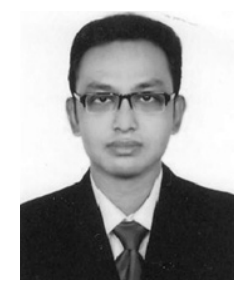

Ahmed Munaz received his B.Sc degree in Electrical \& Electronic Engineering from Ahsanullah University of Science and Technology (AUST), Dhaka, Bangladesh, in 2009. $\mathrm{He}$ is now working as an M.E. candidate at the School of Electrical Engineering, University of Ulsan, South Korea. His research interests are Energy harvesting including power processing circuits and thin film.

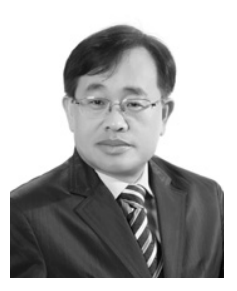

Gwiy-Sang Chung received his B.E. and M.E. degrees in Electrical Engineering from Youngman University, Kyongsan, South Korea, in 1983 and 1985, respectively, and his $\mathrm{Ph}$. D. degree from Toyohashi University of Technology, Toyohashi, Japan, in 1992. He joined ETRI, Daejeon, South Korea in 1992, where he worked on Si-on-insulator materials and devices. In addition, he worked as a visiting scholar at UC Berkeley, and Stanford University, CA, USA, in 2004 and 2009, respectively. He is now working as a professor at the School of Electrical Engineering, University of Ulsan, South Korea. His research interests include $\mathrm{SiC}, \mathrm{ZnO}, \mathrm{AlN}$ M/NEMS, graphene and energy harvesting 